\title{
Onset of unsteadiness in aero-engine intakes at incidence
}

\author{
A. Coschignano* and H. Babinsky ${ }^{\dagger}$ \\ Department of Engineering, University of Cambridge, Cambridge, CB2 1PZ, UK \\ C. Sheaf $f^{\ddagger}$ and E. Platt $\ddagger$ \\ Installation Aerodynamics, Rolls Royce Plc., Derby, DE24 8BJ, UK
}

The flow field around a transonic engine inlet lip at high incidence is investigated for a variety of flow conditions around the design point. Generally, the flow on the upper surface of the lip is characterised by a supersonic region, terminated by a near-normal shock wave. At the nominal design point, the shock is not strong enough to cause significant flow separation for each of the shapes investigated. Off-design conditions were explored by altering the angle of attack as well as changing the mass flow rate over the upper lip, intended to mimic a greater mass flow demand by a turbofan engine. The results suggest that angle of attack is the dominant parameter, where an even relatively small increase of $2^{\circ}$ can lead to large and highly unsteady flow separation with an associated shock oscillation. This is a consequence of the significantly stronger shock compared to the on design case. Both qualitative and quantitative measurements suggest a noticeably reduced aerodynamic performance resulting from higher incidence operation. In contrast, an increase of up to $5.2 \%$ in mass flow did not result in large separated regions or flowfield unsteadiness. However, a trend of increasing separation with greater mass flow was observed.

\section{Nomenclature}

$\begin{array}{ll}\alpha & \text { Angle of incidence } \\ \beta & \text { LDV emitting head angle } \\ \delta & \text { Boundary layer thickness } \\ \dot{m} & \text { Mass flow } \\ U & \text { Flow velocity } \\ M & \text { Mach number } \\ P & \text { Static pressure } \\ I_{\%} & \text { Free-stream turbulence } \\ T & \text { Static temperature } \\ \text { LDV } & \text { Laser Doppler velocimetry } \\ \text { PSP } & \text { Pressure sensitive paint } \\ \text { FFT } & \text { Fast Fourier transform } \\ S u b s c r i p t \\ 0 & \text { Property upstream of the shock - Stagnation value } \\ 1 & \text { Wind tunnel entry property } \\ l & \text { lower channel, usually referred to mass flows } \\ u & \text { upper channel, usually referred to mass flows }\end{array}$

${ }^{*} \mathrm{PhD}$ Student, Department of Engineering, University of Cambridge, AIAA Student Member.

${ }^{\dagger}$ Professor of Aerodynamics, Department of Engineering, University of Cambridge, AIAA Associate Fellow.

$\ddagger$ Installation Aerodynamics, Rolls Royce Plc, Derby. 


\section{Shock-Boundary Layer Interactions in Subsonic Engine Intakes}

When operating under high-thrust conditions, such as during take-off and climb, the substantial mass flow demand by a turbofan engine is sufficient to accelerate the flow over the intake lip to supersonic conditions. This faster-than sound flow pocket is terminated by a near-normal shock wave. The adverse pressure gradient imposed by this disturbance on the boundary layer can cause the latter to separate, introducing large scale unsteadiness and an increase in viscous losses 1 These losses have a direct negative repercussion on the overall engine efficiency and operability as the total pressure reaching the fan face is reduced. Moreover, if the separated boundary layer does not reattach before the engine face, the unsteadiness, characteristic of separated flows, may increase the stress on the fan, which can ultimately reduce component lives and aerodynamic stability margin.

Although a significant amount of research has gone into reducing the detrimental effects associated with shock-induced separation in transonic flight, the majority of these efforts have been limited to aerofoil design. In fact, the formation of shock waves on the inside lip of engine intakes has often been overlooked and thus there is insufficient understanding and a lack of data for CFD validation. To address this shortcoming, Cambridge University has started experimental research into this problem. The first stage of this research investigated the flow field occurring during typical take-off or climb conditions and found the shock-boundary interaction to be relatively benign,${ }^{2]}$ with separated flow confined to small regions of space. However, aeroengine intakes are designed to operate over a wide range, defined by inflow speed, incidence and engine demand. The consequences of increasing the last two parameters are considered in the current investigation. In particular, the main interest is to assess the onset and severity of any unsteady regimes.

A schematic depiction of what the flow topology looks like, for both on and off-design scenarios, is given in Figure 1 .

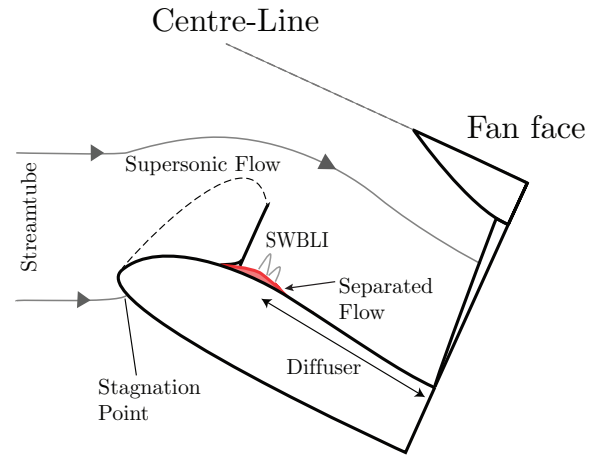

On-design

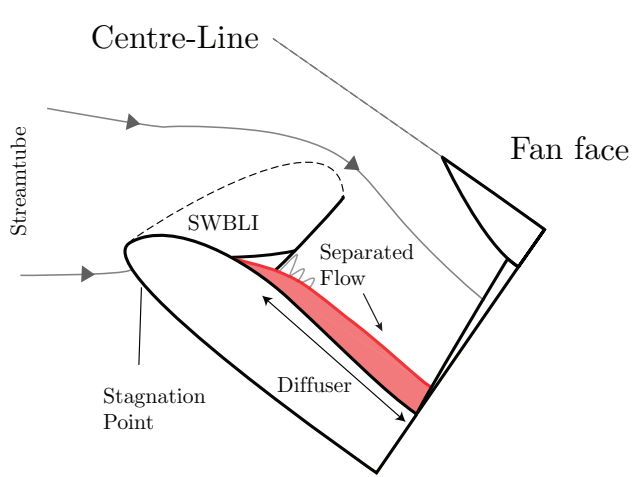

Off-design

Figure 1. Schematic representation of two flow scenarios characterised by different degrees of shock-induced boundary layer separation over an intake lip cross-section during high-incidence flight. Off-design operation is generally associated with a greater degree of flow separation and large scale unsteadiness.

\section{Experimental set-up and methodology}

\section{A. Experimental facility}

All experiments are performed in the high-speed aerodynamics laboratory at the University of Cambridge. In particular, a blow-down wind tunnel, powered by two $50 \mathrm{~kW}$ compressors, is used. The flow is fed from the compressors into the settling chamber, where it is passed through a number of flow straighteners and turbulence grids before a 18:1 contraction. In the current configuration, no nozzle is used and the entry velocity is varied by changing the stagnation pressure and the effective area of the second throat where the flow is chocked. By altering the cross-sectional area of the second throat by means of an aerofoil (see Figure 2) the overall mass flow rate is adjusted, controlling the entry Mach number with a resolution of \pm 0.001 . The working section was designed by Makun ${ }^{3}$ exclusively for the investigation of shock wave boundary layer 
interactions in engine intakes. The working section is depicted in Figure 2 and features curved upper and lower walls, forming a stream-tube divided by a $1 / 7^{\text {th }}$ scale intake lip model. The stream-tube design is based on computational results performed for a real intake. ${ }^{3}$ The upper boundary is a streamline of the computed flow far enough from the supersonic region to avoid choking in the upper channel. The mass flow rate into the lower channel is controlled by choking the flow with an adjustable plug as indicated in Figure 2 The highest supply pressure possible is 2.4 bar. ${ }^{3}$ The experimental operating range is portrayed in Figure 3. The rig is capable of achieving entry conditions of $\mathrm{M}_{1}=0 \rightarrow 0.45$, for $\alpha$ up to 29 degrees. The characteristic length used for calculating the Reynolds number is the maximum intake lip thickness. In the range $\mathrm{M}_{1}=$ $0.25 \rightarrow 0.45$, the experiment is capable of matching full scale Reynolds numbers for altitudes greater than $5000 \mathrm{ft}$ and $25000 \mathrm{ft}$ for a small and large engine respectively. For a given stagnation pressure, by manually reducing the lower channel area, more mass flow is forced into the upper channel mimicking a greater mass flow demand by the engine. As the ratio between the upper and lower channel mass flows increases, the stagnation streamline is lowered, resulting in stronger acceleration and thus a greater supersonic region. This allows the assessment of performance at off-design conditions.

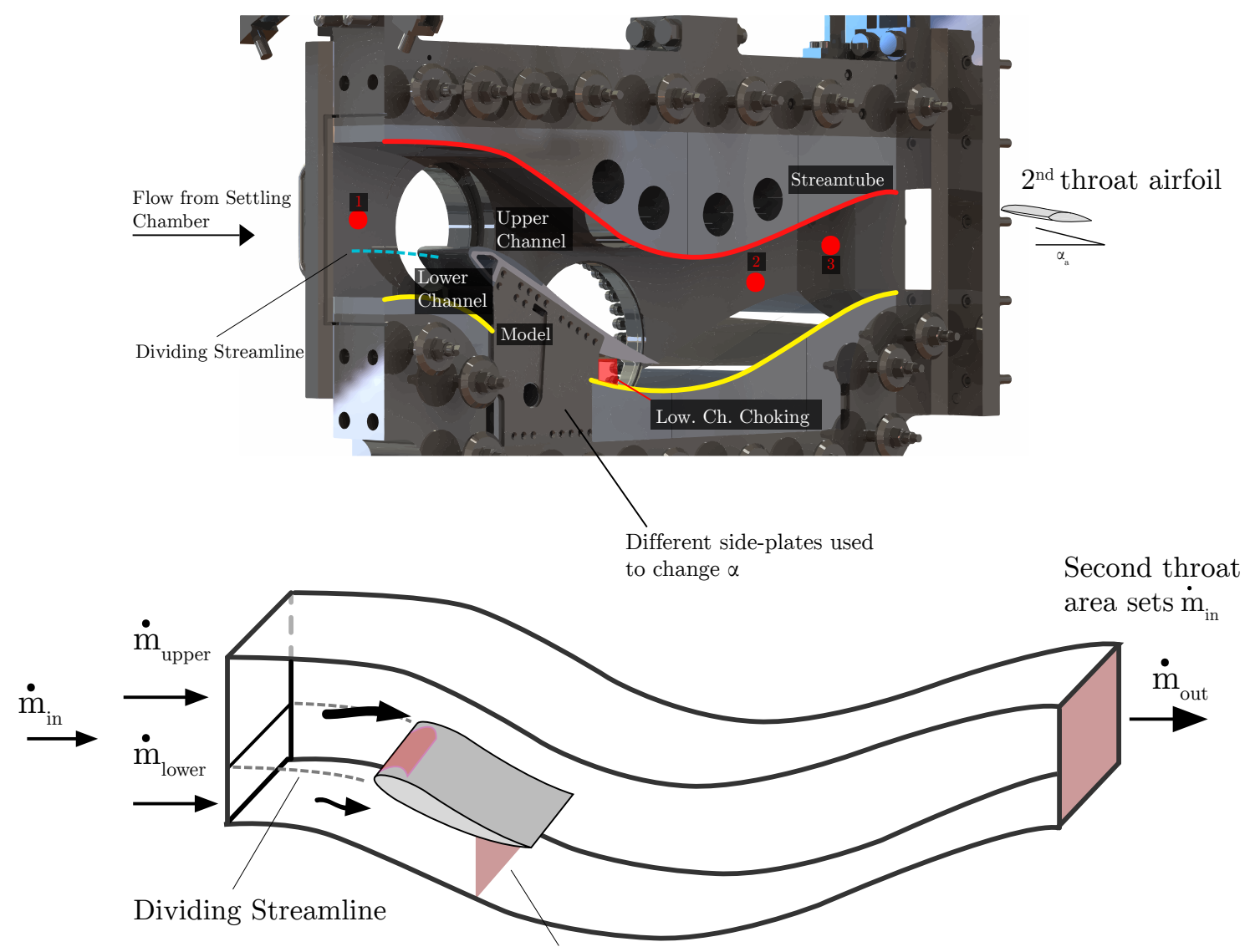

Adjustable plug to choke the channel.

Sets the ratio $\dot{\mathrm{m}}_{\mathrm{u}} / \dot{\mathrm{m}}_{1}$

Figure 2. Representation of the blow-down wind tunnel working section. Stream-tube design based on computed flow streamlines. Reducing the lower channel area by means of a plug allows an adjustment of the mass flow balance between the upper and lower channel.

The settings listed in Table 1 result in a flow field closely matching the target flow provided by both experiments and Rolls Royce computational efforts based on a real intake travelling at a free-stream Mach number of 0.35 and a representative angle of attack, typical for take-off conditions. Section III.B discusses the effects of increased mass flow demand by the engine. This is replicated by keeping the overall mass flow constant while increasing the ratio between upper and lower channel. For on-design investigation, 


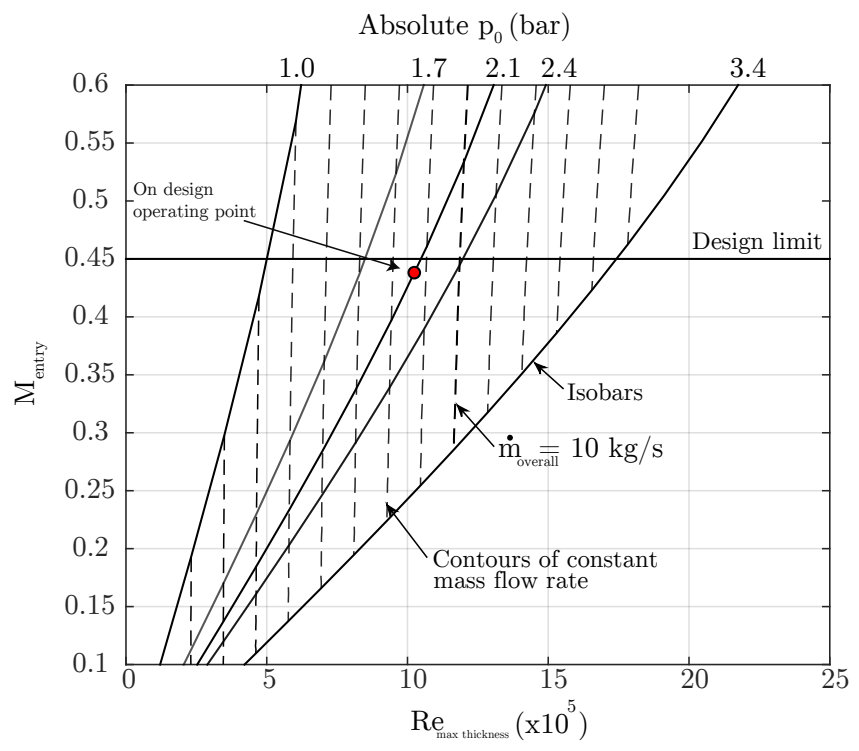

Figure 3. Wind tunnel operational envelope in terms of entry Mach number (or equivalently mass flow rate). Adapted from Makuni. 3

Table 1. Tunnel entry conditions for on-design investigation

\begin{tabular}{cccccccc}
$\dot{m}(\mathrm{~kg} / \mathrm{s})$ & $M_{1}$ & $\alpha($ deg. $)$ & $P_{0}(\mathrm{kPa})$ & $T_{0}(\mathrm{~K})$ & $I_{\%}$ & $\frac{m_{u}}{\dot{m}_{l}}$ & $R e$ \\
\hline 8.68 & 0.435 & 23 & 211.6 & $290 \pm 4$ & 0.88 & 3.78 & $10^{6}$ \\
\hline
\end{tabular}

approximately $26.5 \%$ of the overall mass flow rate is passed through the lower channel. This is reduced in subsequent steps to a minimum of $22.5 \%$, which results in a $5.16 \%$ increase in the upper channel mass flow. Furthermore, by using a different side plate pair, the angle of incidence is increased and the results discussed in Section III.A.

A number of different techniques are used to investigate the flow. In particular, a Schlieren technique is used to visualize the features typical of supersonic flows such as shock waves, as well as expansion and compression waves 4

Surface pressure measurements are taken during the run to both characterise the flow and to assess experimental repeatability. These measurements are obtained by using pressure taps connected via tubing to a differential pressure transducer. Though small in diameter, the presence of a cavity leads to a complex flow field developing over the tap, resulting in a minor over-prediction of static pressure by approximately $0.5 \%$ at worst for the current configuration $\sqrt[5] 6]{6}$ The accuracy of the transducer is rated at $\pm 0.05 \%$.

Furthermore, a number of these pressure readings are used to calibrate pressure sensitive paint. The photons reflected when the latter is excited by UV light are directly proportional to the pressure acting on it. This is technique particularly suited for moderate to high Mach numbers given the higher pressure gradients involved. The Mach number on the surface considered ranges from $M=0.7$ to $M=1.8$. This is high enough to provide reliable measurements ${ }^{7}$ An optical device is used to measure such luminescence, resulting in a number of pressure readings equivalent to the camera resolution available. The relationship between luminescence intensity and pressure is determined by the Stern-Volmer relation: $\frac{8}{8}$

$$
\frac{I_{\text {ref }}}{I_{(P, T)}}=A(T)+B(T) \frac{P}{P_{r e f}}
$$

$I_{r e f}$ is the luminescence taken at a reference pressure and temperature. The obvious choice is to measure the intensity at normal atmospheric conditions with the tunnel off. A second image is taken with the flow on. A no flow - no light background image is subtracted to both images to reduce noise from external light sources. Finally, accordingly to the Stern-Volmer equation, the flow-off reference image is divided by the 
flow-on image to obtain the pressure ratio. This also helps reducing the impact of thermal effects on the accuracy of PSP. In addition, large temperature changes along the surface are prevented as a result of the test model being manufactured with an almost constant thickness, By using the pressure taps located on the model, the values of the constants $A(T)$ and $B(T)$ can be determined and the absolute pressure values can be extracted from raw light intensity. The overall error associated with pressure sensitive paint can be evaluated by observing the mean deviation of the static pressure measurements from the calibration curve. This is found to be ranging from approximately $2 \%$ to a maximum of $4 \%$. To minimise the calibration error 5-6 points are required ${ }^{8}$ Given the rig configuration and the position of the access windows, the images are captured at an angle and are, thus, characterised by severe perspective distortion. Therefore, all PSP images have been processed with an un-wrapping algorithm to permit the presentation of 2-dimensional contour plots.

In addition to pressure taps, the model is also equipped with four flush-mounted fast-response pressure transducers. Their accuracy is estimated to be $0.5 \%$ of the full scale signal.

Flow velocities are measured using a two component Laser Doppler Velocimetry (LDV) system. Two pairs of coherent laser beams, with a wavelength of $561 \mathrm{~nm}$ and $532 \mathrm{~nm}$ respectively, are focused inside the working section to form the interference pattern of the ellipsoidal working volume, measuring $130 \mu \mathrm{m}$ in diameter. Kerosene particles, with a diameter of approximately $0.5 \mu \mathrm{m}^{5}$, are used to seed the flow and allow velocity measurements to be recorded via a proprietary software. The laser emitting head and receiving optics are mounted on a traverse capable of moving in one direction with a user defined velocity. The signal is sampled at an optimised variable rate to exploit a full signal cycle leading to a typical measurement accuracy, as stated by the manufacturer, of $\pm 0.1 \%$ of $U_{\max }(\sim 580 \mathrm{~m} / \mathrm{s})$. In addition the emitting head is oriented at an angle $\beta=8.5^{\circ}$ to allow the surface to be reached by the incident beams. This angle yields a small error in the vertical velocity component just above $1 \%$. The horizontal velocity component is, on the other hand, unaffected by $\beta$. Proper seeding in the area of interest is crucial to maximise accuracy. This is considerably high in the free-stream but drops as the wall is approached. As a result, considering the aforementioned sources of error, the overall deviance of the measured values from the real one is estimated to be below \pm $2 \%$. However, this is higher in the proximity of the wall, within the inner-most portion of he boundary layer.

Stagnation temperature is recorded by using 4 T-type thermocouples placed in the settling chamber. A linear drop of stagnation temperature from $\sim 294 \mathrm{~K}$ to $\sim 286 \mathrm{~K}$ is observed during an average 30 second long run. This variation in stagnation temperature is taken into account when converting absolute velocity measurements from LDV to local Mach number to minimise the error involved, which would otherwise peak $1.7 \%$.

\section{Results}

\section{A. Baseline flow topology and increasing incidence}

The effect of increasing the angle of incidence from the reference $23^{\circ}$ to $25^{\circ}$, while keeping the overall mass flow constant, as well as the ratio between $\dot{m}_{u}$ and $\dot{m}_{l}$, is hereby reported. This is aimed to replicate a steeper operation of the intake while travelling at the same inflow Mach number and with the engine demand kept to the same value.

Figure 4 a shows Schlieren photograph of the baseline flow. The flow topology is typical for a high thrust climb condition, described in more detail in a previous paper. ${ }^{2}$ Due to the pronounced incidence, the flow undergoes a strong acceleration around the lip. A pocket of supersonic flow is created near the intake highlight, which is then terminated by a normal shock wave occurring at approximately $12 \%$ of the chord. From qualitative observations alone, the interaction of this shock with the incoming boundary layer appears to be relatively benign. No $\lambda$ structure is evident, suggesting small or absent shock-induced separation. Velocity measurement, obtained by means of LDV and shown in Figure 5 , indicate the Mach number at the shock foot to be around 1.4, with the flow having already undergone a brief isentropic deceleration from a slightly higher peak Mach number greater than $\mathrm{M}=1.5$. This compression is most likely due to geometry effect and pressure gradient due to curvature on the lip. Nonetheless, at the shock foot, the Mach number is beyond the commonly accepted limit for shock-induced separation. Oil flow visualization confirmed the presence of a small recirculating region, showing a small owl face type separation. ${ }^{2}$ However, this separation is not large enough to cause any significant adverse effects.

When the incidence is increased to 25 degrees, however, the flow starts to break down. A $\lambda$ structure is now clearly distinguishable in Figure $4 \mathrm{p}$, suggesting a more severe shock-induced separation. The flow

5 of 12

American Institute of Aeronautics and Astronautics 
re-acceleration over this flow region causes a moderate supersonic tongue to be formed downstream of the normal shock, signified by the presence of small secondary shocklets. At this flow condition the peak Mach number ahead of the shock is $\mathrm{M} \approx 1.6$ which is well above the level expected to cause flow separation. Similar to the baseline flow, there is a small amount of isentropic compression ahead of the shock as seen in Figure 5. Velocity measurements in the upstream boundary layer have been attempted unsuccessfully as the size of the LDV measurement volume is of the same order of magnitude as the estimated $\delta_{i}$. However, given the substantial pressure jump and the flow topology, both interactions are believed to be turbulent in nature, with the transition occurring close to the lip highlight.

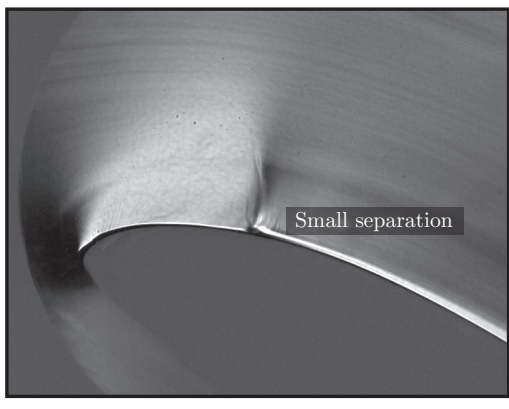

a) Baseline, $23^{\circ}$

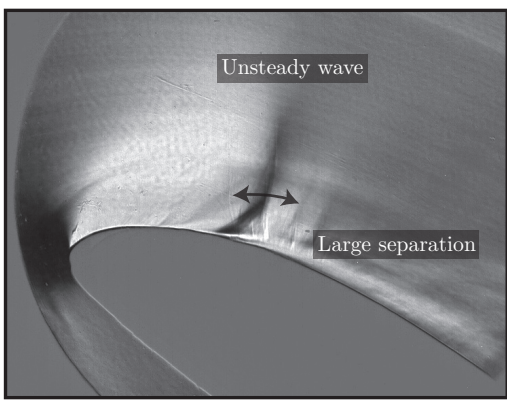

b) $25^{\circ}$

Figure 4. High speed Schlieren photograph comparison for increasing angle of incidence.

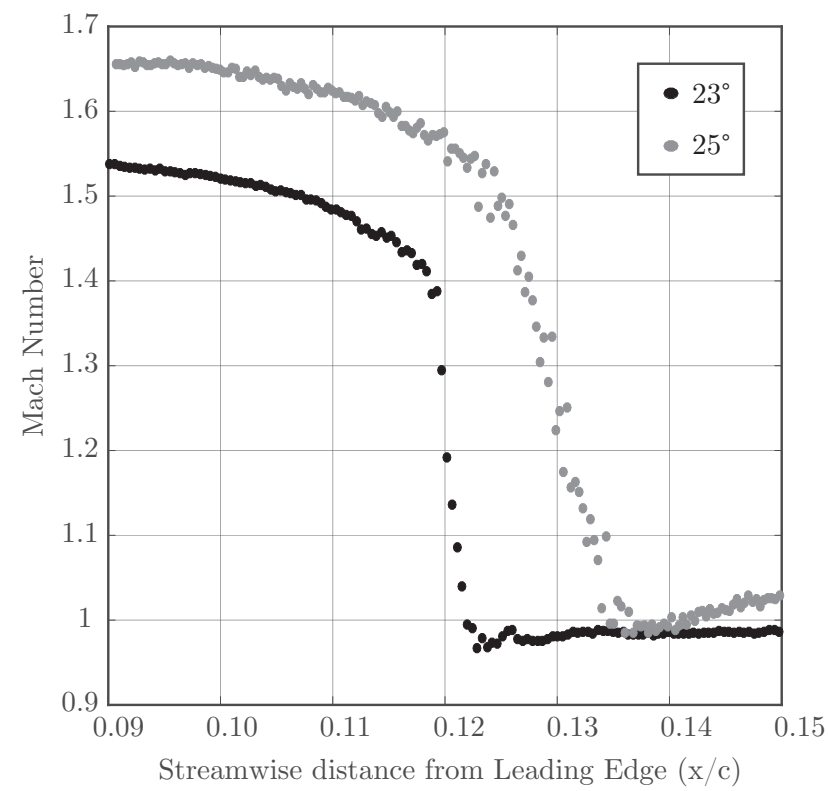

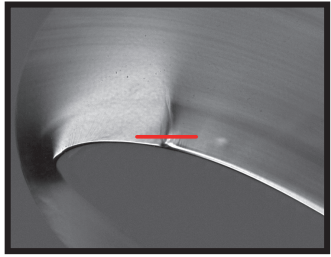

a) $23^{\circ}$

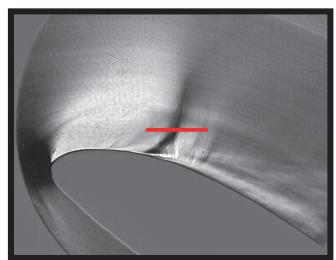

b) $25^{\circ}$

Figure 5. Streamwise traverse across the inviscid region of the shock wave at the locations indicated by the red line. Higher incidence results in greater acceleration upstream of the interaction and consequently stronger shock wave.

The stream-wise velocity measurements depicted in Figure 5 show a more smeared velocity change across the shock-wave in the higher incidence case when compared to the reference case. To investigate whether this could be due to shock unsteadiness high-speed Schlieren video was employed. The shock location was tracked by image processing to an accuracy of a few pixels $(\approx 0.5 \mathrm{~mm})$ with a sampling rate of $4 \mathrm{kHz}$. Figure 6 shows the fast Fourier transform spectrum of the shock motion. It can be seen that the most dominant frequencies are confined to low frequencies in the range $100 \mathrm{~Hz}-300 \mathrm{~Hz}$.

The first peak is noticeable at around $101 \mathrm{~Hz}$ with two harmonics at around $200 \mathrm{HZ}$ and $300 \mathrm{HZ}$ respectively. At higher incidence, in the $100-300 \mathrm{~Hz}$ band the wave oscillation amplitude levels are one order of magnitude 

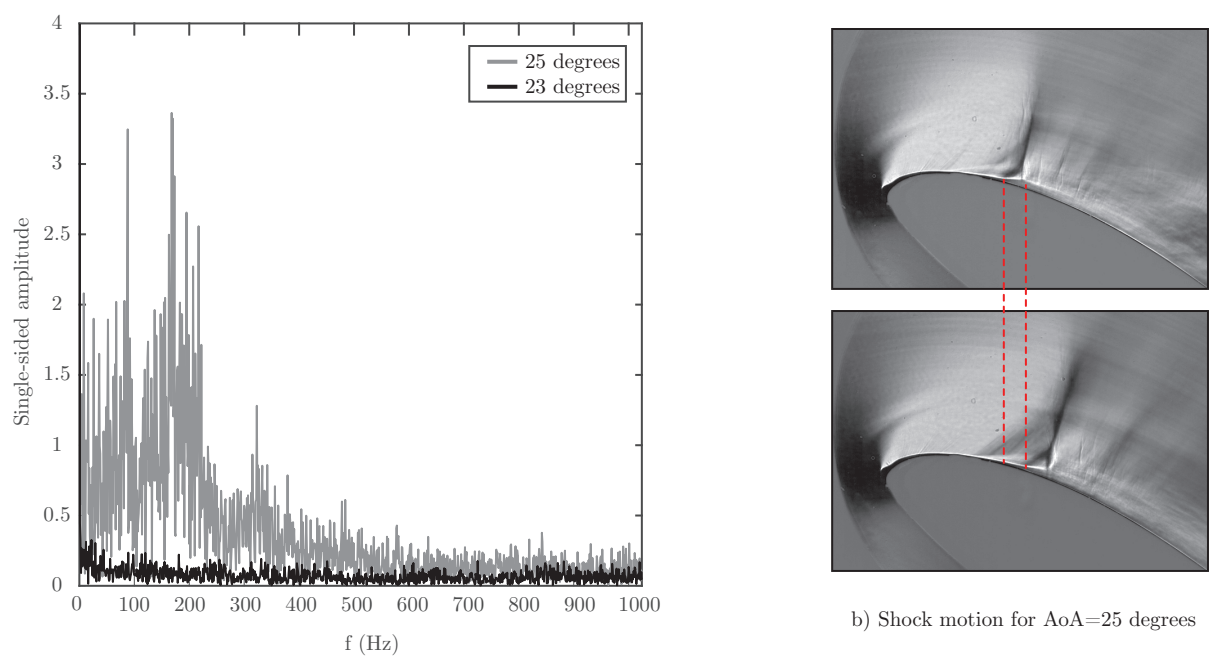

b) Shock motion for $\mathrm{AoA}=25$ degrees

Figure 6. Single-sided amplitude spectrum of the shock-wave position from high-speed Schlieren analysis. Relative amplitude of the wave motion is significantly larger for the $25^{\circ}$ incidence. Nonetheless, no clear dominant frequency is found. Schlieren images on the right provide a visualization of the approximate shockmotion range.

larger than the on-design reference flow. The adverse pressure gradients due to geometry definition and more severe incidence do not favour flow reattachment and the separated bubble grows pushing the shock upstream. The large amplitude-low frequency oscillation is likely caused by a feedback mechanism between the shock wave and the separation bubble similar to transonic buffeting on wings.

The highest r.m.s recorded by flush-mounted surface pressure transducers reached $14 \%$ of the mean immediately upstream of the shock.

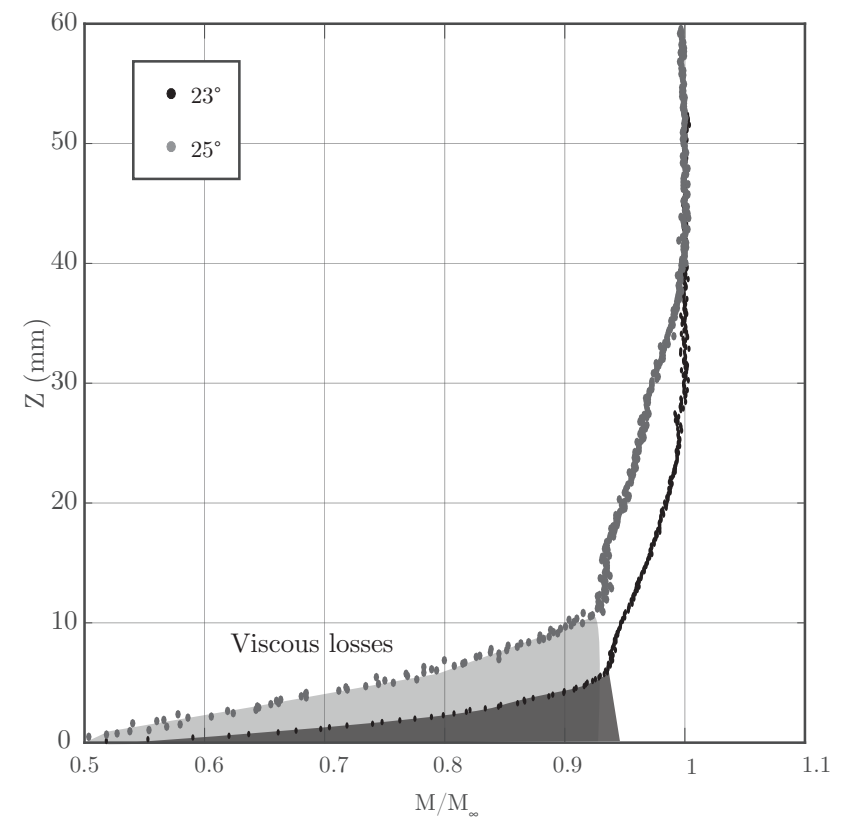

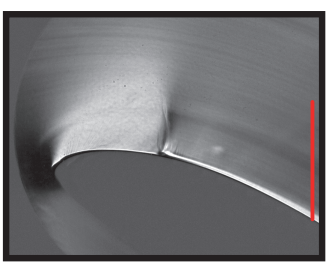

a) $23^{\circ}$

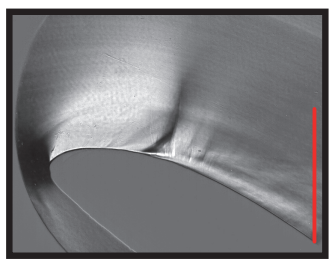

b) $25^{\circ}$

Figure 7. Normal to ground velocity measurements downstream of the interaction. Higher incidence (grey) shows a thicker viscous layer and noticeably less full profile in the outer region due to increased wave drag.

Figure 7 shows velocity distributions recorded downstream of the SBLI, close to the virtual engine plane, 
indicated by the orthogonal red line in Figure 7. It can be seen that the off-design flowfield exhibits a significantly increased boundary layer thickness close to the wall. Further away from the surface, the greater shock strength seen in the off-design case is indicated by reduced Mach number extending further into the flowfield. This is consistent with the other measurements and imply a lower pressure recovery at greater incidence.

\section{B. Increasing "Engine Demand"}

As explained in the methodology section, the minimum area of the lower channel can be reduced by means of an adjustable plug. By keeping the ratio between the tunnel entry area and the second throat constant the entry mass flow is maintained to the on-design value $(8.68 \mathrm{~kg} / \mathrm{s})$. For the reference configuration, $6.39 \mathrm{~kg} / \mathrm{s}$ are discharged via the upper channel. The following section examines the resulting flow topology when this is increased up to $6.72 \mathrm{~kg} / \mathrm{s}$ (i.e: a $5.16 \%$ increase) in three, equally spaced steps, controlling the stagnation streamline position. This effectively provides a way to replicate an increased demand by the engine that may occur during take off or high incidence climb and manoeuvring. This investigation has been extended to both incidence levels considered so far. Starting point for both configurations is the mass flow ratio defined in Table 1 and assessed in Section III A

A first, qualitative, depiction of the flow field is given in Figure 8, showing Schlieren photographs for four different upper channel mass flow values. These images clearly indicate that the two incidence levels respond differently to changes in mass flow ratio.

For the more benign $23^{\circ}$ case, as the mass flow in the upper channel increases, the shock wave moves further downstream and the supersonic region grows significantly. With increasing shock strength the interaction domain grows and eventually a clear $\lambda$ structure emerges indicating flow separation. Nonetheless, the size of the lambda, even for the greatest mass flow considered, is relatively small, suggesting that the separation remains of modest extent.

Contrarily, as shown in Figure 8 second row, it would appear that raising the angle of attack results in a reduced sensitivity to flow rate surge in the upper channel. In fact, the already unsteady and separated flow topology shows no noticeable change.

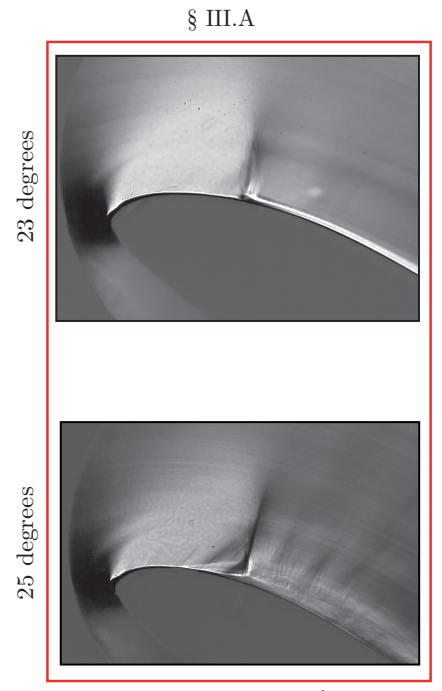

a) On design $\dot{\mathrm{m}}$
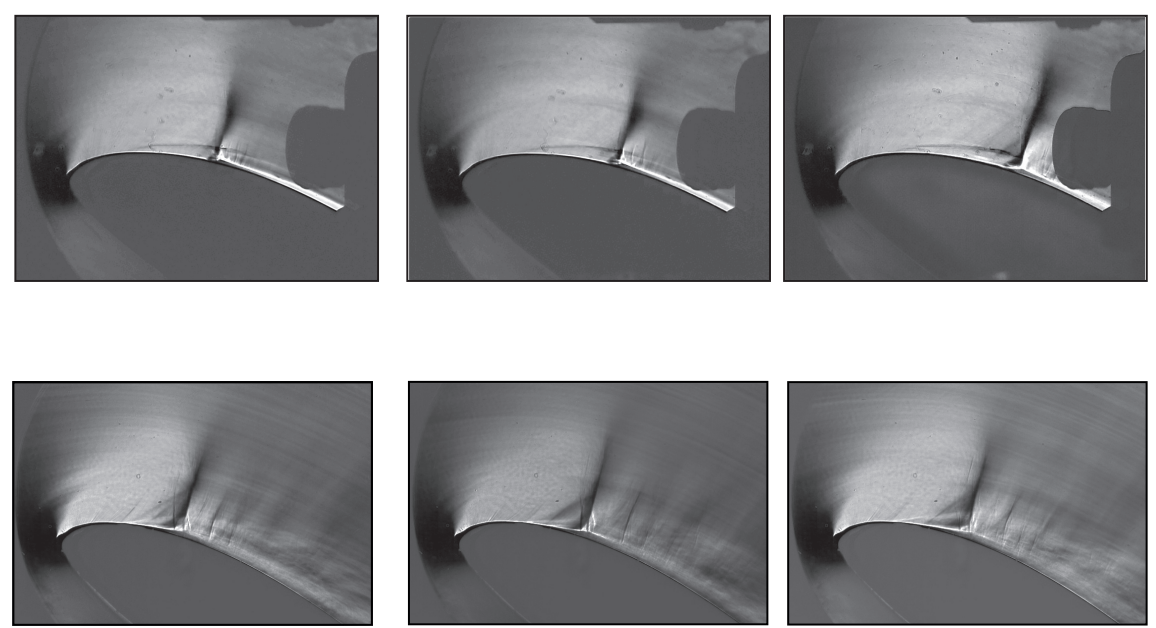

b) $+1.72 \% \dot{\mathrm{m}}_{\mathrm{u}}$

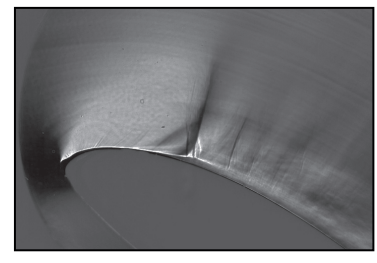

c) $+3.44 \% \dot{\mathrm{m}}$

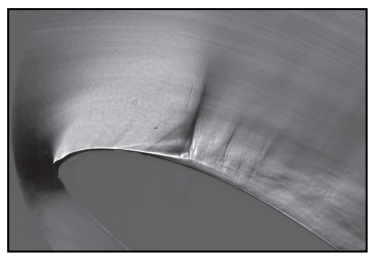

d) $+5.16 \% \dot{\mathrm{m}}_{\mathrm{u}}$

Figure 8. Schlieren photographs captured by means of a high-speed camera for the two angle of incidence. Note very little change for the higher incidence (bottom).

Supporting quantitative evidence is provided in Figure 9a. Horizontal LDV traverses recorded across the shock for $\alpha=23$ deg clearly show its change in position and strength with mass flow rate. In contrast, the results at the larger angle of attack show little variation in shock strength or location. 
a) 23 degrees

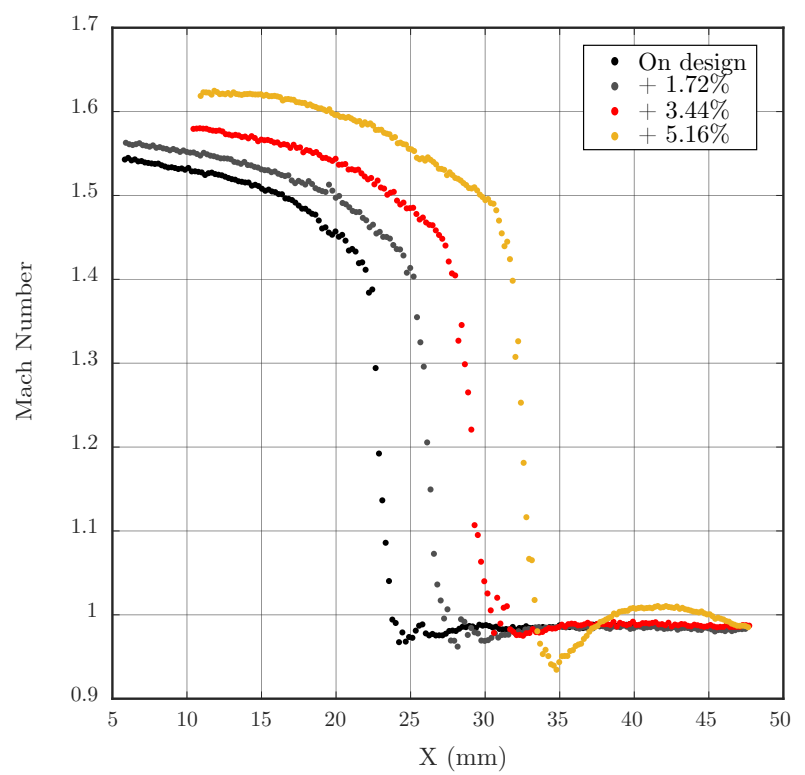

b) 25 degrees

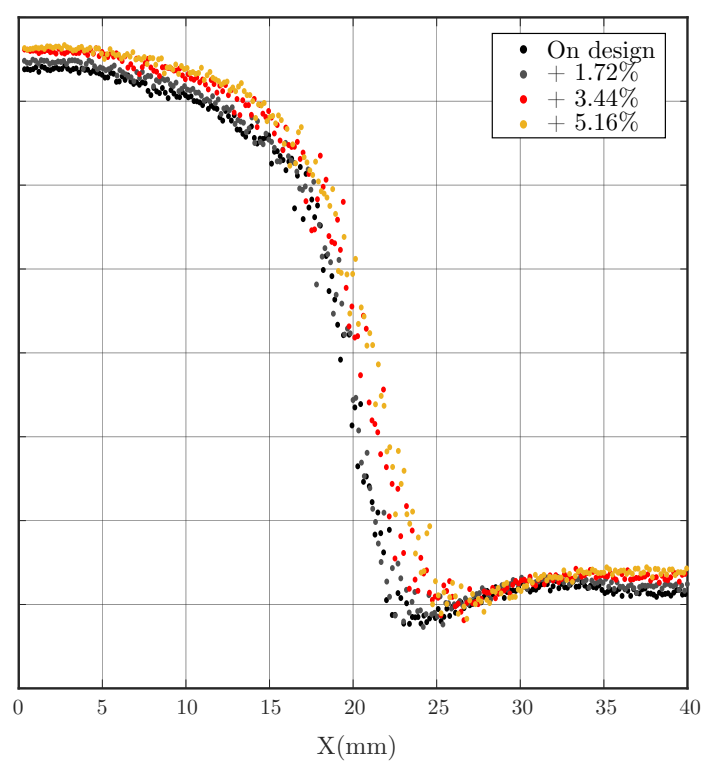

Figure 9. Series of stream-wise velocity measurements across the inviscid region of the shock-wave for the two incidence configurations across the range of upper channel mass flows.

Figure 10 presents the isentropic surface Mach number as determined from PSP. It can be seen that corner effects are confined to a small region with the flow being practically two-dimensional in the centre span. The solid line indicates an isentropic Mach number of unity. Note that there are substantial supersonic regions behind the shock suggesting the presence of a sizeable supersonic tongue. The overall size of the interaction, as measured from the upstream influence location (where the isentropic Mach number first decreases) to the sonic line is a rough guide to the separation size. For the lower angle of attack the sonic line remains close to the main shock across the whole mass flow range considered, suggesting that any separation is relatively small. This does not appear to vary in size with increasing mass flow rate. On the other hand, at the increased $\alpha=25$ degrees, although shock strength and location appear, consistently with LDV data, practically unvaried, the interaction length appears to grow in size. This might be an indication of the flow approaching complete breakdown. If this happens, the shock is expected to move upstream as a result of the separation bubble bursting. It is possible that, just before full breakdown occurs, the shock remains roughly in the same location as it is just about to reverse its motion.

As seen in Figure11, changes in shock oscillation amplitude (as recorded from high-speed Schieren video) has been investigated to determine the impact of increasing demand on shock motion and onset of unsteadiness. Neither configuration showed significant changes in unsteadiness amplitude across the range taken into consideration for the current paper. In fact, the FFT spectra obtained by tracking the shock-wave position show no significant growth in amplitude with mass flow ratio. The lower incidence remained relatively benign. Similarly, the already moderately unsteady flow encountered at higher incidence, shows no noticeable variation in oscillation amplitude. These observations are based on the spectra reports in Figure

Unsteady pressure measurements along the surface show a mild rise in fluctuation intensity with increasing mass flow (Figure 12p. This is thought to be due to the small shift in the position of the shock relative to the measurement points. Though the change in position between the extremes of the operation envelope considered was found to be less than $1.5 \mathrm{~mm}$, this is approximately $10 \%$ of the region of space investigated by means of closely spaced fast response pressure transducers. Downstream of the SBLI, the fluctuation intensity values approach the same value for the different conditions. Ultimately, complete flow breakdown was not observed under any circumstance considered. 

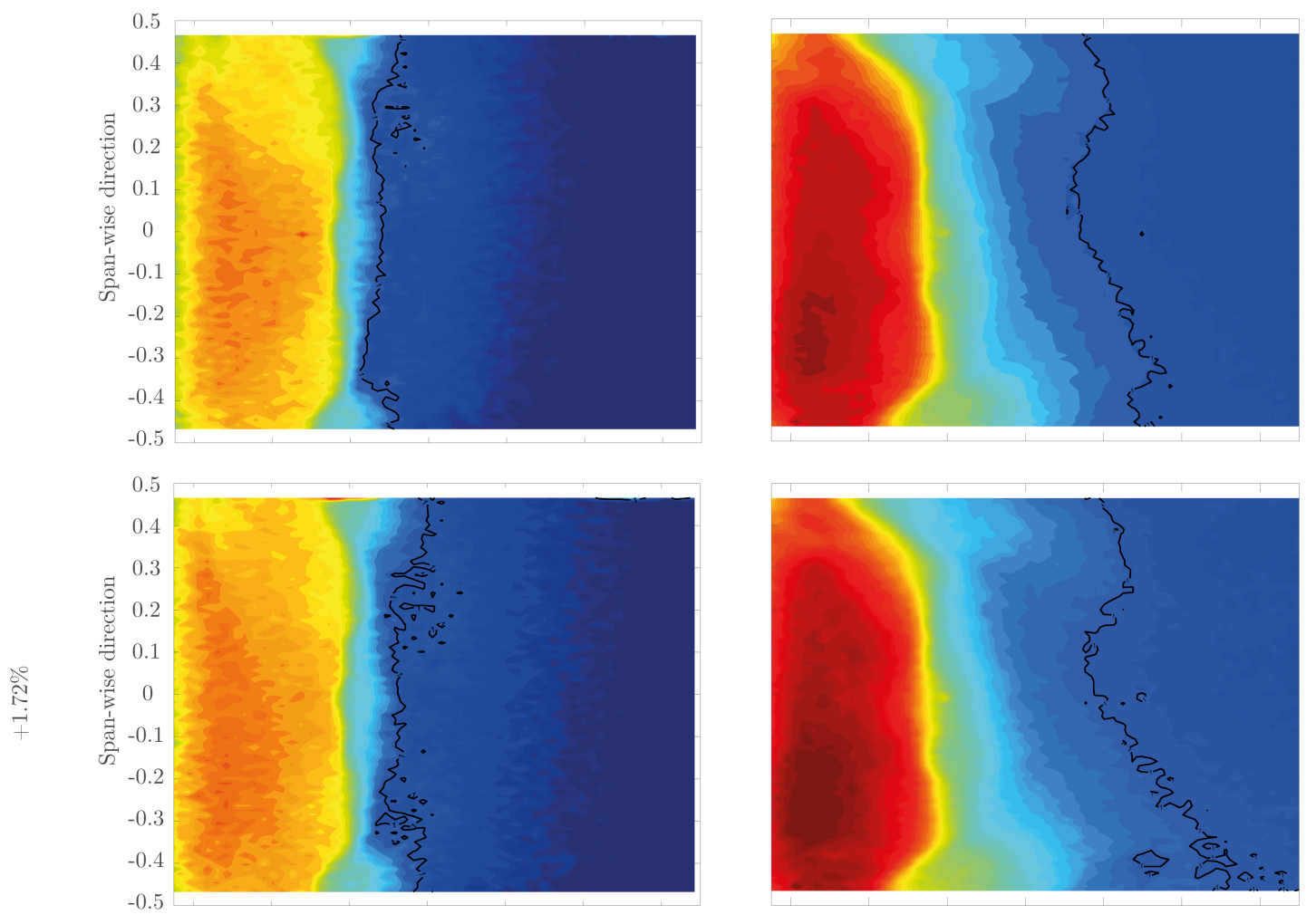

Mach
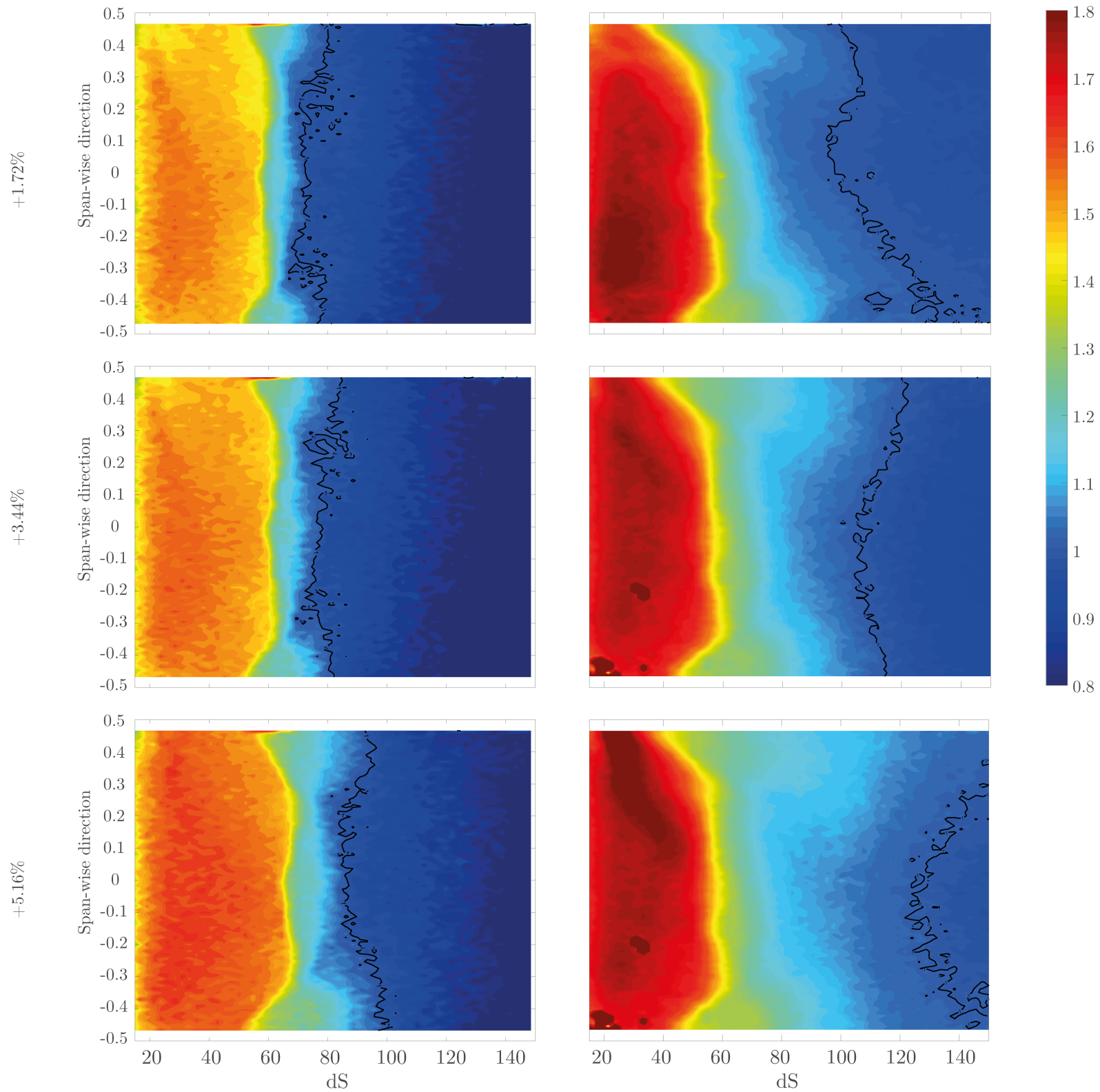

Figure 10. Isentropic Mach number obtained from pressure sensitive paint for increasing mass flow rates. These measurements confirm the reduced sensitivity of the flow to increases in mass flow when the incidence is increased.

$$
10 \text { of } 12
$$



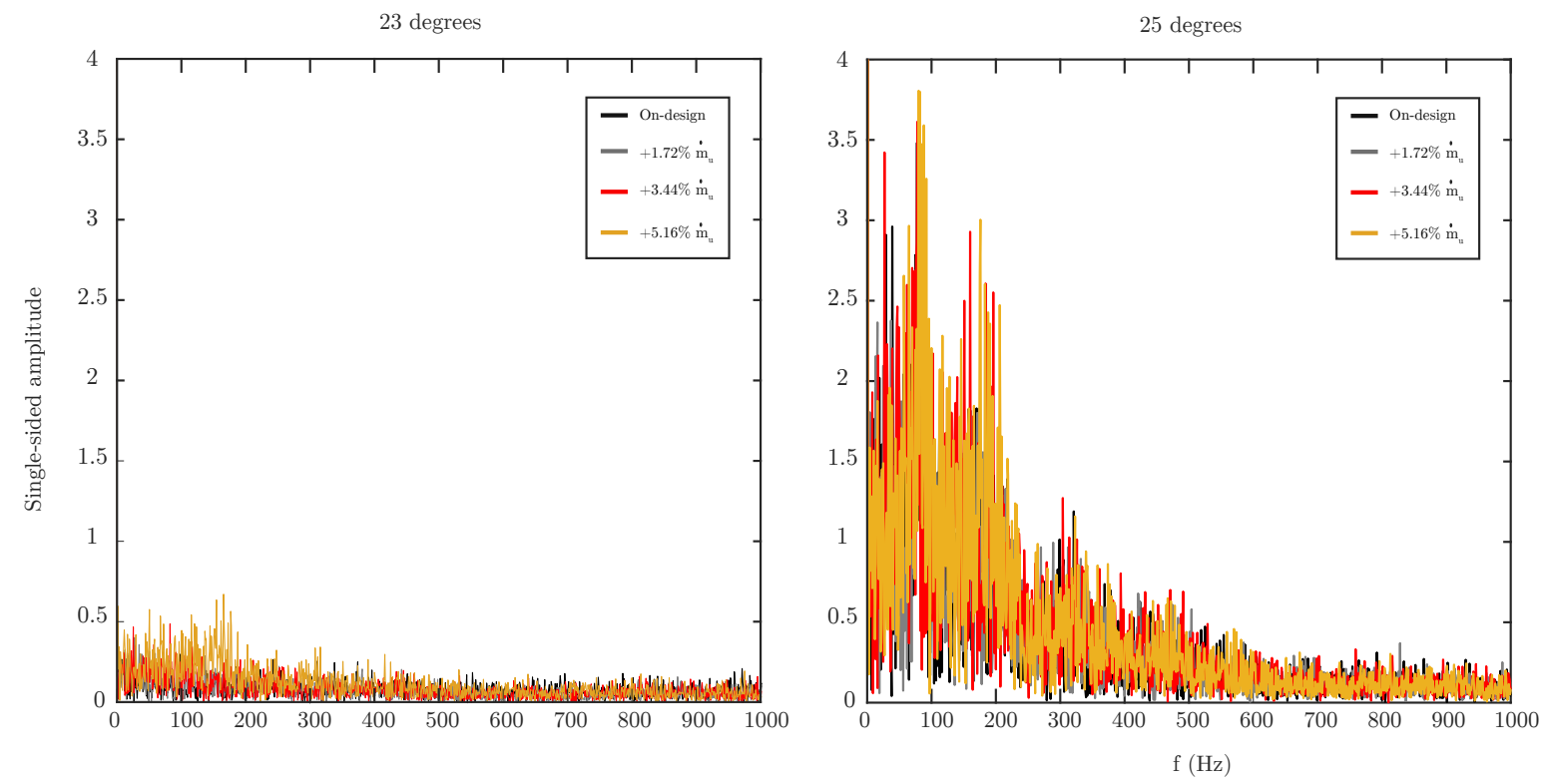

Figure 11. Single side amplitude spectrum of the shock-wave position as obtained from high-speed Schlieren analysis. Overall, the increase in 'reference engine flow' considered appears not to have a major impact on the development of unsteady features.

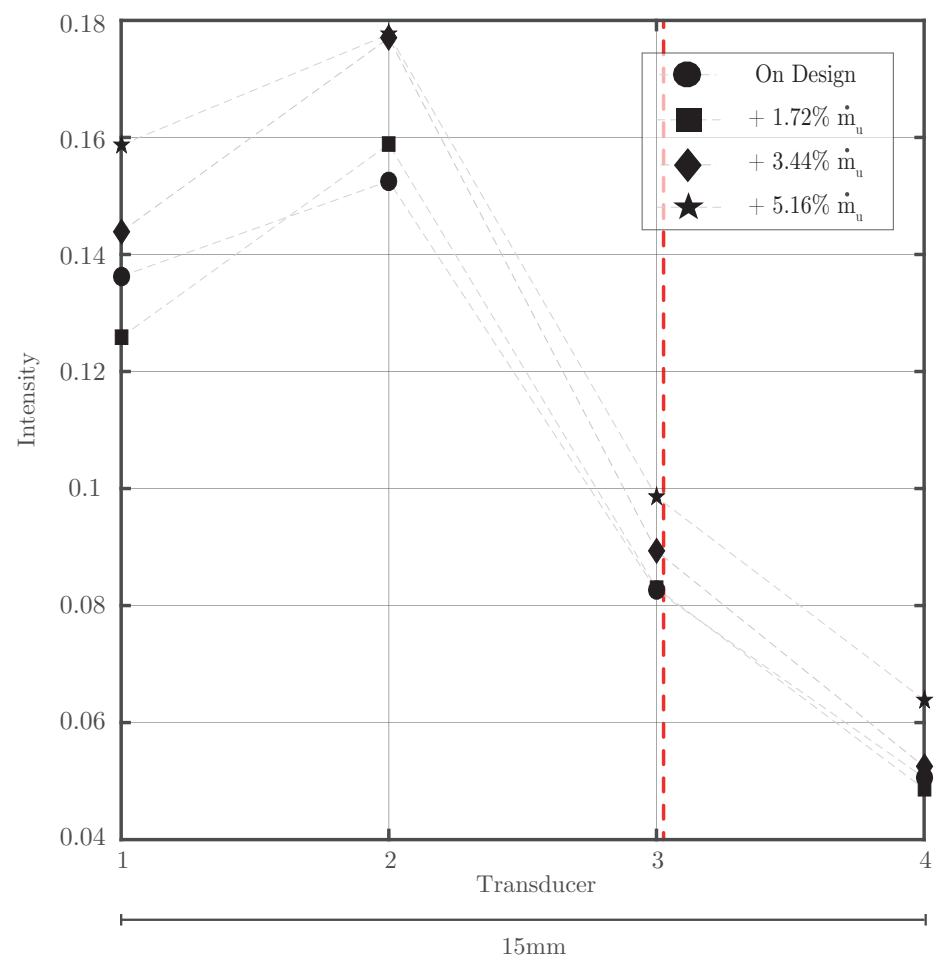

Figure 12. Pressure fluctuation intensity obtained from surface mounted pressure sensors for $\alpha=25$ degrees. The shaded region indicates oscillation range of the shock, observed to be nearly identical for all the mass flow rates considered (difference between most upstream position recorded $<1.2 \mathrm{~mm}$ ). Mean shock position around Tap 3 ( $\pm 1.3 \mathrm{~mm}$ between extreme cases $)$. 


\section{Conclusion}

After matching and characterising the flow topology observed over intake lips during typical high-incidence operation, ${ }^{2}$ the flow conditions and set-up have been modified in order to assess its performance across a wider portion of the operational envelope. In particular, both an increased flow demand from a turbo-fan engine and changing angle of incidence have been replicated and examined in the CUED wind-tunnels. Whereas the reference flow, defined by an incidence of 23 degrees and moderate engine demand, is relatively benign in nature, with only minor flow recirculation, a modest incidence increase by 2 degrees(to $\left.25^{\circ}\right)$ shows a noticeably greater degree of shock-induced separation and led to the onset of moderate unsteadiness. As a result, the viscous losses downstream are considerably larger, compromising the pressure recovery capabilities of the intake. On the other hand, increasing 'engine demand' had a dissimilar effect at different incidence levels. For the lowest angle considered $\left(23^{\circ}\right)$, as the flow over the upper channel increases, the shock moves proportionally downstream and its strength grows. The steeper incidence, on the other hand, showed a reduced sensitivity to mass flow ratio surges. In fact, no major growth in unsteadiness amplitude, nor shift of the mean shock location, were reported across the range considered. This is unexpected but may be a sign that the flow is approaching breakdown. Supporting evidence is found in the continuous growth of the separation size downstream of the normal shock despite no change in its strength. Overall, complete breakdown was not observed across the envelope considered. Nonetheless, the evidence collected so far suggests that increases in angle of incidence have a greater effect on the growth of separation and onset of flow unsteadiness than surges in engine mass flow rate. This is thought to be caused by the more rapid growth of shock strength with angle of attack compared to changes in mass flow.

\section{Acknowledgements}

The authors wish to acknowledge David Martin, Sam Flint, Anthony Luckett and John Hazlewood for operating the CUED blow-down wind tunnel and Kevin Bullman for the manufacturing of the models used in this investigation. Moreover, they would like to thank Rolls Royce Plc and the Engineering and Physical Sciences Research Council (EPSRC) for funding the current research.

\section{References}

\footnotetext{
${ }^{1}$ BABINSKY, H. and HARVEY, J. K., Shock-wave-Boundary layer Interactions, Cambridge University Press, 2011.

${ }^{2}$ COSCHIGNANO, A., BABINSKY, H., SHEAF, C., and PLATT, E., "Impact of near-leading edge curvature on the performance of aero-engine intakes at incidence," 16th AIAA Aviation Technology, Integration, and Operations Conference, 2016.

${ }^{3}$ MAKUNI, T. E., BABINSKY, H., SLABY, M., and SHEAF, C. T., "Shock Wave-Boundary-Layer Interactions in Subsonic Intakes at High Incidence," 53rd AIAA Aerospace Sciences Meeting. January, 2015.

${ }^{4}$ FERRI, A., Elements of Aerodynamics of Supersonic Flows, Macmillan Co., 1949.

${ }^{5}$ COLLISS, S. P., Vortical structures on three-dimensional shock control bumps, PhD Thesis, University of Cambridge, 2014.

${ }^{6}$ MEIER, H., "Measuring techniques for compressible turbulent boundary layers," NASA STI/Recon Technical Report N, Vol. 79, Nov. 1977.

${ }^{7}$ Sajben, M., "Uncertainty estimates for pressure sensitive paint measurements," AIAA Journal, Vol. 31, No. 11, 1993, pp. 2105-2110.

${ }^{8}$ GREGORY, J. W., ASAI, K., KAMEDA, M., LIU, T., and SULLIVAN, J. P., "A review of pressure-sensitive paint for high-speed and unsteady aerodynamics," Proc. IMechE Vol. 222 Part G: J. Aerospace Engineering, 2007.
} 